Sharif University of Technology
Scientia Iranica
SCIENTIA
I RAN I C A
http://scientiairanica.sharif.edu

\title{
A study on the accuracy of finite volume numerical models with non-rectangular mesh
}

\author{
M. Morovvat ${ }^{a}$, A.R. Zarrati ${ }^{\mathrm{b}, *}$, and M.R. Jalili Ghazizadeh ${ }^{\mathrm{a}}$ \\ a. Department of Water and Environmental Engineering, Shahid Beheshti University, East Vafadar Blvd., Tehranpars, Tehran, \\ P.O. Box 1658953571, Iran. \\ b. Department of Civil and Environmental Engineering, Amirkabir University of Technology, Tehran, P.O. Box 1591634311, Iran.
}

Received 12 October 2018; received in revised form 4 December 2020; accepted 11 January 2021

\author{
KEYWORDS \\ Non-rectangular \\ mesh; \\ Complex geometry; \\ Numerical error; \\ Finite volume method; \\ Velocity oscillation; \\ Discretization \\ assumptions; \\ Differential advection.
}

\begin{abstract}
In the process of numerical simulation of complex geometries and flow depth variations, non-rectangular computational cells are generated. However, the application of this type of mesh causes numerical errors. A 3D model, which was verified and validated before, was used to shed light on the problem in a simple, open channel flow. A zigzag computational mesh was used to study the effect of non-rectangular cells on the accuracy of the model. Results showed that water surface and velocity profiles oscillated around the correct answer. Investigating the reason for this oscillation showed that assuming constant velocity on non-rectangular computational cell surfaces, which is a usual practice in all numerical schemes, cause this error. Variation of velocity on mesh surfaces was then added to the computation model and as a result, the oscillations in velocity profiles and water surface were eliminated. Further analysis showed that this is a general problem in any finite volume method with a non-rectangular mesh.
\end{abstract}

(C) 2021 Sharif University of Technology. All rights reserved.

\section{Introduction}

Analysis of flow in natural streams and complex channel systems using numerical models has become very popular in the last three decades [1-12]. Dealing with a complex geometry of flow boundaries and complicated bed topography as in rivers is among the many problems that numerical modelers may face [1315]. The computational grid used in such conditions should be generated with a non-rectangular mesh to ensure compliance with irregular boundaries such as the non-orthogonal curvilinear coordinate systems, sigma

\footnotetext{
*. Corresponding author. Tel.: 009821 64543002;

Fax: 00982166414213

E-mail addresses: m_morovvat@yahoo.com (M. Morovvat);

Zarrati@aut.ac.ir (A.R.Zarrati); m_jalili@sbu.ac.ir (M.R. Jalili Ghazizadeh)
}

doi: $10.24200 /$ sci. 2021.52017 .2484 coordinate, etc. [5,7,16-20]. However, discretization of the governing equations around non-rectangular mesh results in numerical errors, especially when mesh shapes are very distorted [16].

In the present work, a multi-layer 3D shallowwater model, which was developed and calibrated before [17], is tested with non-rectangular computational cells to show the computational errors with such a mesh. The results of the model are compared with an analytical solution and numerical errors are analyzed. In the following sections, the employed model is introduced first and then, the problem with a non-rectangular mesh and how it can be solved are demonstrated and discussed. The final section demonstrates that the same error can occur for any finite volume model.

\subsection{The employed model}

For simplicity, a 3D shallow-water model is employed 
here. In these models, pressure in depth is assumed hydrostatic and the water column is divided into a number of layers similar to computational cells that share friction terms and convective fluxes. The shallow water equations are then integrated over the thickness of each layer as is usual in any finite volume model. Multi-layer models are primarily used with horizontal and parallel layers to solve coastal flows [21-25] and flow in open channels $[3,4,6]$. To increase the flexibility in complex topographies, a generalized multi-layer model with non-parallel layers was developed [17]. In fact, with non-parallel layers, computational mesh cells could be trapezoidal in depth. In the present work, it was intended to study the effect of non-rectangular computational cells on the accuracy of the model by comparing the results with analytic solutions. It will be shown later that the results of shallow water equations are also applicable to and valid for any finite volume solution of the equations.

\subsection{Governing equations}

Equations for 3-D shallow water flow in a steady state condition for an incompressible fluid neglecting wind stresses and Coriolis effects are:

$$
\begin{aligned}
& \frac{\partial u}{\partial x}+\frac{\partial v}{\partial y}+\frac{\partial w}{\partial z}=0, \\
& \frac{\partial}{\partial x}\left(u^{2}\right)+\frac{\partial}{\partial y}(u v)+\frac{\partial}{\partial z}(u w)=-\frac{1}{\rho} \frac{\partial P}{\partial x}-g_{x} \\
& \quad+\frac{\partial}{\partial x}\left(v_{t} \frac{\partial u}{\partial x}\right)+\frac{\partial}{\partial y}\left(v_{t} \frac{\partial u}{\partial y}\right)+\frac{\partial}{\partial z}\left(v_{t} \frac{\partial u}{\partial z}\right), \\
& \frac{\partial}{\partial x}(v u)+\frac{\partial}{\partial y}\left(v^{2}\right)+\frac{\partial}{\partial z}(v w)=-\frac{1}{\rho} \frac{\partial P}{\partial y}-g_{y} \\
& \quad+\frac{\partial}{\partial x}\left(v_{t} \frac{\partial v}{\partial x}\right)+\frac{\partial}{\partial y}\left(v_{t} \frac{\partial v}{\partial y}\right)+\frac{\partial}{\partial z}\left(v_{t} \frac{\partial v}{\partial z}\right), \\
& -\frac{1}{\rho} \frac{\partial P}{\partial z}=g_{z},
\end{aligned}
$$

where $u, v$, and $w$ are velocity components in the $x$, $y$ and $z$ directions, respectively; $P$ is pressure; $\rho$ is density; $g_{x}, g_{y}$, and $g_{z}$ are the components of gravitational acceleration in each of the three directions, and $v_{t}$ is eddy viscosity. First, Eq. (4) is substituted into Eqs. (2) and (3) and is, then, integrated over the $k$ th layer. In this integration, a control volume is assumed with upper and lower surfaces inclined in both of the $x$ and $y$ directions (Figure 1 ). Leibnitz rule is used in this integration as follows [17]:

$$
\int_{z_{k}}^{z_{k}+1} \frac{\partial f}{\partial k} d z=\frac{\partial}{\partial k} \int_{z_{k}}^{z_{k}+1} f d z-\left[f \frac{\partial z}{\partial k}\right]_{z_{k}}^{z_{k+1}},
$$

where $z_{k}$ and $z_{k+1}$ are the elevations of bottom and top surfaces of the $k$ th layer, respectively, relative to

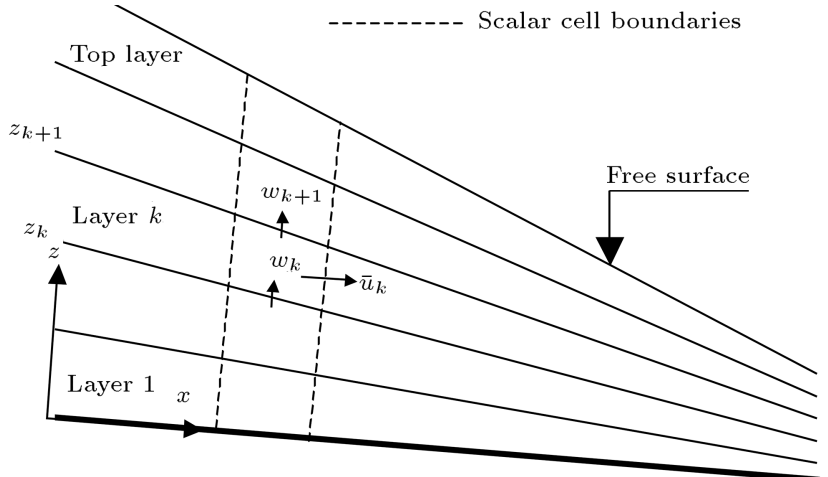

Figure 1. Layout of the multi-layer model and location of variables.

bed and along the $z$ axis. Following the process of integration, the governing equations are derived as follows [17]:

$$
\begin{aligned}
\frac{\partial}{\partial x}\left(\bar{u} h_{k}\right)+ & \frac{\partial}{\partial y}\left(\bar{v} h_{k}\right)+\left.M\right|_{z_{k}}-\left.M\right|_{z_{k+1}}=0 \\
\frac{\partial}{\partial x}\left(\bar{u}^{2} h_{k}\right)+ & \frac{\partial}{\partial y}\left(\overline{u v} h_{k}\right)=-g h_{k} \frac{\partial \zeta}{\partial x} \\
& +\frac{\partial}{\partial x}\left[v_{t} \frac{\partial\left(\bar{u} h_{k}\right)}{\partial x}\right]+\frac{\partial}{\partial y}\left[v_{t} \frac{\partial\left(\bar{u} h_{k}\right)}{\partial y}\right] \\
& +\left.S r^{u}\right|_{z_{k}}-\left.S r^{u}\right|_{z_{k+1}}, \\
\frac{\partial}{\partial x}\left(\overline{v u} h_{k}\right)+ & \frac{\partial}{\partial y}\left(\bar{v}^{2} h_{k}\right)=-g h_{k} \frac{\partial \zeta}{\partial y} \\
& +\frac{\partial}{\partial x}\left[v_{t} \frac{\partial\left(\bar{v} h_{k}\right)}{\partial x}\right]+\frac{\partial}{\partial y}\left[v_{t} \frac{\partial\left(\bar{v} h_{k}\right)}{\partial y}\right] \\
& +\left.S r^{v}\right|_{z_{k}}-\left.S r^{v}\right|_{z_{k+1}},
\end{aligned}
$$

where:

$$
\begin{aligned}
& M=u \frac{\partial z}{\partial x}+v \frac{\partial z}{\partial y}-w \\
& S r^{u}=v_{t}\left(2 \frac{\partial u}{\partial x} \frac{\partial z}{\partial x}+2 \frac{\partial u}{\partial y} \frac{\partial z}{\partial y}-\frac{\partial u}{\partial z}\right)-M u \\
& S r^{v}=v_{t}\left(2 \frac{\partial v}{\partial x} \frac{\partial z}{\partial x}+2 \frac{\partial v}{\partial y} \frac{\partial z}{\partial y}-\frac{\partial v}{\partial z}\right)-M v .
\end{aligned}
$$

In these equations, $\zeta$ is the elevation of water surface; $h_{k}$ the thickness of the $k$ th layer; and $\bar{u}$ and $\bar{v}$ are layeraveraged velocities in the center portion of each layer as follows:

$$
\bar{u}=\frac{1}{h_{k}} \int_{z_{k}}^{z_{k+1}} u d z, \quad \bar{v}=\frac{1}{h_{k}} \int_{z_{k}}^{z_{k+1}} v d z,
$$

where $\partial z / \partial x$ and $\partial z / \partial y$ are the slopes of the layer interfaces in the $x$ and $y$ directions, respectively. In multi-layer models, the terms $M, S r^{u}$, and $S r^{v}$ denote 
the mutual effects of different layers on each other. The term $M$ is the mass flux across the layer interfaces. It is obvious that $M$ is zero on the water surface and channel bed. The terms in the brackets in $S r^{u}$ and $S r^{v}$ show shear stresses at the interface. Shear stresses at the lower interface of the bottom layer (that is the channel invert) result from bed friction. There is no shear stress on the water surface since wind stress is neglected. $M u$ and $M v$ are the convective fluxes due to mass transfer between the layers.

During the above-mentioned analysis, the following terms are neglected from the left hand side of Eqs. (7) and (8), respectively:

$$
\begin{aligned}
& \frac{\partial}{\partial x} \int_{z_{k}}^{z_{k+1}}(u-\bar{u})(u-\bar{u}) d z, \quad \frac{\partial}{\partial y} \int_{z_{k}}^{z_{k+1}}(u-\bar{u})(v-\bar{v}) d z, \\
& \frac{\partial}{\partial x} \int_{z_{k}}^{z_{k+1}}(u-\bar{u})(v-\bar{v}) d z, \quad \frac{\partial}{\partial y} \int_{z_{k}}^{z_{k+1}}(v-\bar{v})(v-\bar{v}) d z .
\end{aligned}
$$

These terms are called differential advection and are produced from the integration of advection terms over layers' thickness (similar to integration of equations over a computational cell in a finite volume model). These terms originate from the differences in the averaged velocity in each layer and the actual velocity distribution in that layer and describe a lateral momentum exchange [26]. Given that velocity distribution over a layer is not known, differential advection terms are usually neglected $[3,21,24]$; or, in other words, velocity is assumed constant in a layer. This is similar to assuming constant velocity on cell faces when integration is conducted over a computational cell using the finite volume method.

\subsection{Discretization method and the solution algorithm}

Eqs. (7) and (8) are discretized by integration over the computational cells. Checkerboard pressure oscillation was avoided employing a staggered grid [27]. Central differencing scheme and hybrid scheme were used to discretize the diffusion and convection terms, respectively. The discretized momentum equations are written as follows [17]:

$$
\begin{aligned}
a_{P}^{u} \bar{u}_{P}= & \sum_{n b=W, E, S, N} a_{n b}^{u} \bar{u}_{n b}+S r^{u} \Delta x \Delta y \\
& -g h_{k} \frac{\partial \zeta}{\partial x} \Delta x \Delta y, \\
a_{P}^{v} \bar{v}_{P}= & \sum_{n b=W, E, S, N} a_{n b}^{v} \bar{v}_{n b}+S r^{v} \Delta x \Delta y \\
& -g h_{k} \frac{\partial \zeta}{\partial y} \Delta x \Delta y,
\end{aligned}
$$

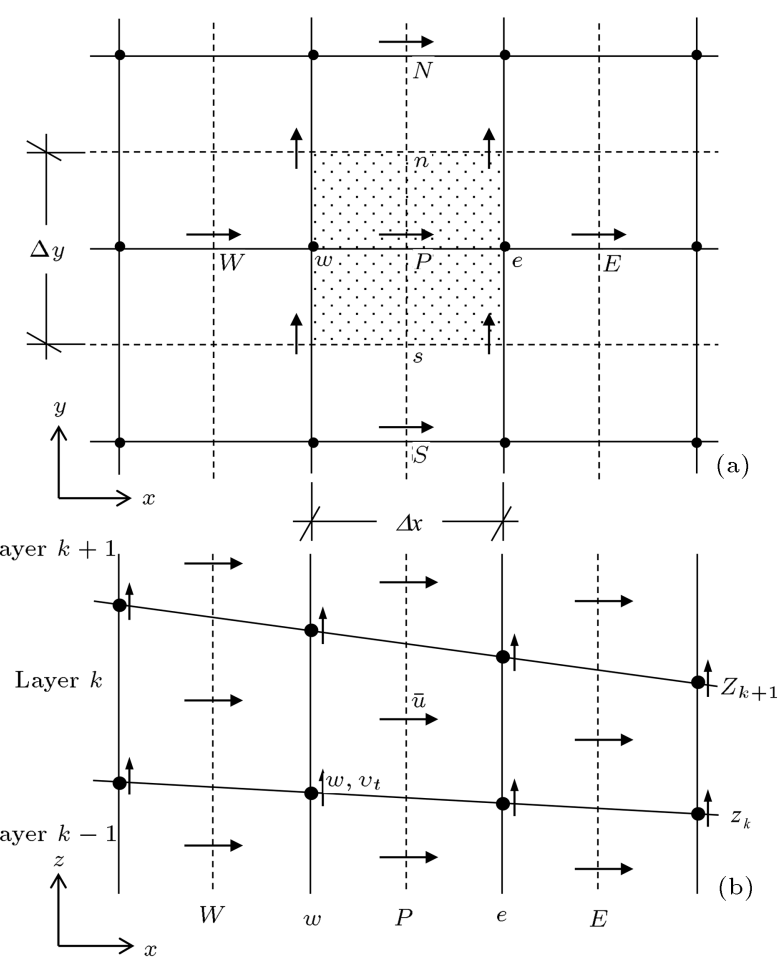

Figure 2. Computational grid and location of variables in (a) plan and (b) depth.

where $\Delta x$ and $\Delta y$ are the mesh dimensions in plan; $P$ is the center of the control volumes $\bar{u}$ or $\bar{v}$, and $W, E, S$, and $N$ are the grid points surrounding it (Figure 2(a)); superscripts $u$ and $v$ denote the momentum equation in the $x$ and $y$ directions, respectively; and $S r^{u}$ and $S r^{v}$ are the source terms that include the discretized state of all the terms given in Eqs. (10) and (11). These terms are calculated at each grid point by interpolating the values of the variables neighboring it from the last iteration (Figure 2(b)); $a$ is the coefficient of discretization and $a_{P}$ is as follows:

$$
\begin{aligned}
a_{P}^{u, v}= & a_{W}^{u, v}+a_{E}^{u, v}+a_{S}^{u, v}+a_{N}^{u, v} \\
& +\left(F_{e}^{u, v}-F_{w}^{u, v}+F_{n}^{u, v}-F_{s}^{u, v}\right),
\end{aligned}
$$

where:

$$
F_{e}^{u, v}-F_{w}^{u, v}+F_{n}^{u, v}-F_{s}^{u, v}=\left(\left.M^{u, v}\right|_{z_{k+1}}-\left.M^{u, v}\right|_{z_{k}}\right) \Delta x \Delta y \text {. }
$$

The above equation is the discretized continuity equation (Eq. (6)) and $F$ terms are mass fluxes in and out of a cell face in the $x$ and $y$ directions.

In an iterative manner considering boundary condition, the discretized momentum equations are solved for each layer to get the final converged results. From this solution, the layer averaged velocity components in $x$ and $y$ directions are determined at all grid points. However, these velocities are based on velocities and flow depth from the previous iteration and they may 
not satisfy the continuity equation, which needs to be corrected $[28,29]$. For coupling depth and velocity fields, an equation should also be derived for depth correction [17]. Given that the values of the velocity components and depth in each iteration are shown by an asterisk, the required correction is represented by a prime sign, and $h$ is total depth, we have:

$$
\bar{u}=\bar{u}^{*}+\bar{u}^{\prime}, \quad \bar{v}=\bar{v}^{*}+\bar{v}^{\prime}, \quad h=h^{*}+h^{\prime} .
$$

The thickness of all layers in the present model does not vary in the calculation procedure, except the top one; therefore, it can be written as follows:

$$
\zeta=\zeta^{*}+\zeta^{\prime}=\zeta^{*}+h^{\prime}
$$

According to Eq. (19), in each iteration, Eq. (15) can be written as follows:

$$
a_{P}^{u} \bar{u}_{P}^{*}=\sum_{n b=W, E, S, N} a_{n b}^{u} \bar{u}_{n b}^{*}+S r^{u} \Delta x \Delta y-g h_{k} \frac{\partial \zeta^{*}}{\partial x} \Delta x \Delta y .
$$

Subtracting the above equation from Eq. (15) results in:

$$
a_{P}^{u} \bar{u}_{P}^{\prime}=\sum_{n b=W, E, S, N} a_{n b}^{u} \bar{u}_{n b}^{\prime}-g h_{k} \frac{\partial \zeta^{\prime}}{\partial x} \Delta x \Delta y .
$$

Following the SIMPLEC algorithm [27] in the $y$ direction yields:

$$
\begin{aligned}
& \bar{u}_{P}=\bar{u}_{P}^{*}-g h_{k}\left(\frac{\partial h^{\prime}}{\partial x}\right) \Delta x \Delta y / C_{e f \bar{u}} \\
& \bar{v}_{P}=\bar{v}_{P}^{*}-g h_{k}\left(\frac{\partial h^{\prime}}{\partial y}\right) \Delta x \Delta y / C_{e f \bar{v}}
\end{aligned}
$$

where $C_{e f \bar{u}}=a_{P}^{u}-\sum a_{n b}^{u}$ and $C_{e f \bar{v}}=a_{P}^{v}-\sum a_{n b}^{v}$. Eqs. (23) and (24) are the velocity correction equations. The continuity equation for the whole flow depth is written below:

$$
\begin{aligned}
& \sum_{k=1}^{k=n l}\left[\left(\bar{u} h_{k}\right)_{e}-\left(\bar{u} h_{k}\right)_{w}\right] \Delta y \\
& \quad+\sum_{k=1}^{k=n l}\left[\left(\bar{v} h_{k}\right)_{n}-\left(\bar{v} h_{k}\right)_{s}\right] \Delta x=0
\end{aligned}
$$

where $n l$ is the number of layers. If Eqs. (23) and (24) are substituted into the discretized continuity equation, an equation for depth correction is resulted as follows:

$$
a_{P} h_{P}^{\prime}=a_{W} h_{W}^{\prime}+a_{E} h_{E}^{\prime}+a_{S} h_{S}^{\prime}+a_{N} h_{N}^{\prime}+S u,
$$

where $S u$ is called mass residual and is the difference between the outflow and inflow of each water column. $S u$ should become zero when the solution is converged.
Therefore, the sum of absolute values of $S u$ over all the columns is the convergence indicator. In addition, we have:

$$
\begin{aligned}
& a_{P}=\sum a_{n b}=a_{W}+a_{E}+a_{S}+a_{N}, \\
& a_{W}=-g \sum_{k=1}^{k=n l}\left[\frac{h_{k}^{2}}{C_{e f \bar{u}}}\right]_{w} \Delta y^{2}, \\
& a_{E}=-g \sum_{k=1}^{k=n l}\left[\frac{h_{k}^{2}}{C_{e f \bar{u}}}\right]_{e} \Delta y^{2}, \\
& a_{S}=-g \sum_{k=1}^{k=n l}\left[\frac{h_{k}^{2}}{C_{e f \bar{v}}}\right]_{s} \Delta x^{2}, \\
& a_{N}=-g \sum_{k=1}^{k=n l}\left[\frac{h_{k}^{2}}{C_{e f \bar{v}}}\right]_{n} \Delta x^{2},
\end{aligned}
$$

where $w, e, s$, and $n$ are positions on the faces of the scalar cells.

Finally, the algorithm of the solution can be summarized as follows [17]:

1. Set all initial and boundary conditions;

2. Solve momentum equations for $\bar{u}$ and $\bar{v}$ (Eqs. (15) and (16)) in the 3D space;

3. Solve the depth correction equation (Eq. (26)) and update depth;

4. Update velocity components based on the depth correction values through Eqs. (23) and (24). The updated values satisfy the continuity equation;

5. Calculate velocity components in the depth direction $w$ using the continuity equation in each layer (Eq. (6)), starting from the bottom layer;

6. Check convergence criterion. If it is not satisfied, repeat Steps 2 to 6.

\subsection{Boundary conditions}

In sub-critical flows, the flow depth at the outlet section is specified and depth gradient is set to zero at the inlet. No boundary condition is necessary for $\bar{u}$ velocities at the outlet [27] and $\partial \bar{v} / \partial x=0$. With the discharge and flow depth at hand, the velocity distribution at the inlet is calculated at each iteration. An appropriate velocity distribution law (for example, logarithmic distribution) should be assumed at the inlet. At solid boundary, velocities normal to the boundary are set to zero. To calculate the boundary shear stress (bed and wall friction), the wall function is employed. Depth correction at all boundaries is set to zero. The initial free surface elevation in the whole flow domain is assumed equal to water surface elevation at the outlet section. The initial values of $\bar{v}$ and $w$ are equal to zero and $\bar{u}$ is set equal to the averaged velocity of the initial section. 


\section{Examining the accuracy of the model with a non-rectangular mesh}

In order to examine the effect of non-rectangular mesh on the accuracy of results, the results of the model with rectangular and non-rectangular meshes were compared in a simple uniform flow. In a uniform flow, velocity in flow direction changes only in depth and other velocity components are zero. It will be much easier to find the source of errors in such a simple flow. If any error was recognized in this simple condition, they would be expected in more complicated flows. For this purpose, a channel with a width of $0.5 \mathrm{~m}$, a length of $5 \mathrm{~m}$, a slope of 0.001 , and absolute bed roughness of $0.007 \mathrm{~m}\left(k_{s}\right)$ was assumed. The side wall friction was neglected. By considering $0.2 \mathrm{~m}^{3} / \mathrm{s}$ discharge, the flow depth in this channel would be $0.4 \mathrm{~m}$.

The velocity distribution calculated by the numerical model was compared with the following analytical equation [17]:

$$
\begin{aligned}
\frac{u}{u_{*}}= & \frac{2}{\kappa}\left(\sqrt{1-z / h}-\ln \frac{1+\sqrt{1-z / h}}{z / h}\right) \\
& +\frac{1}{\kappa} \ln \left(\frac{16.25 h}{k_{s}}\right),
\end{aligned}
$$

where $u$ is the velocity component parallel to the bed, $u_{*}$ the bed shear velocity, $\kappa$ Von Karman constant equal to $0.4, z$ distance measured from the bed, and $h$ depth. This equation is derived by assuming the Prandtl's mixing length equal to $\kappa z$ and a linear distribution for shear stress in depth.

At the first stage, the model was executed with rectangular and fine meshes in plan and depth. Prandtl's mixing length model was applied for eddy viscosity computations, and the mixing length was assumed equal to $\kappa z$, similar to that used in deriving Eq. (28). With the mesh size used, the results of the model were independent of the thickness of the layers and the model resulted the depth of $0.4 \mathrm{~m}$ correctly. Also, the calculated velocity distribution in depth conformed well to Eq. (28).

At the next stage, the effect of non-rectangular computational cells on the model results was examined. For this purpose, a 15-degree zigzag mesh was generated for the assumed uniform flow, as shown in Figure 3. With this mesh, the slope of the top

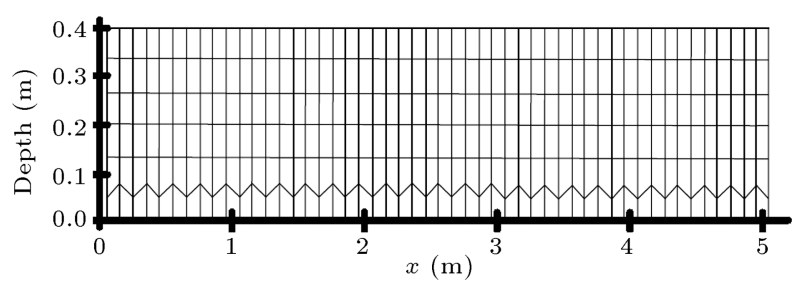

Figure 3. Zigzag mesh in a uniform flow.

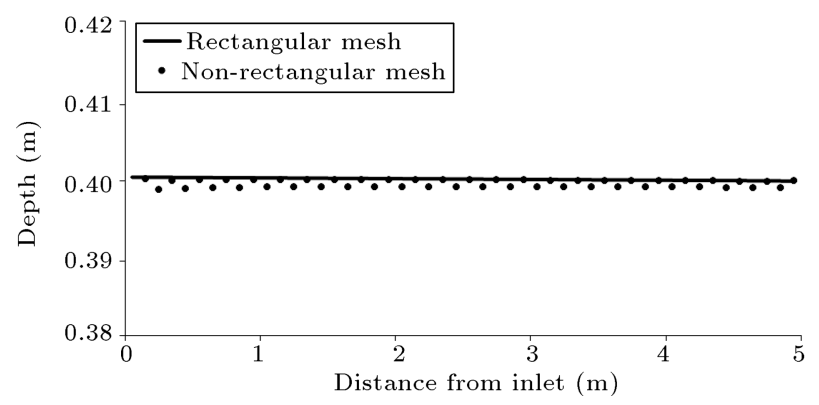

Figure 4. Oscillation of the calculated flow depth in a non-rectangular mesh .

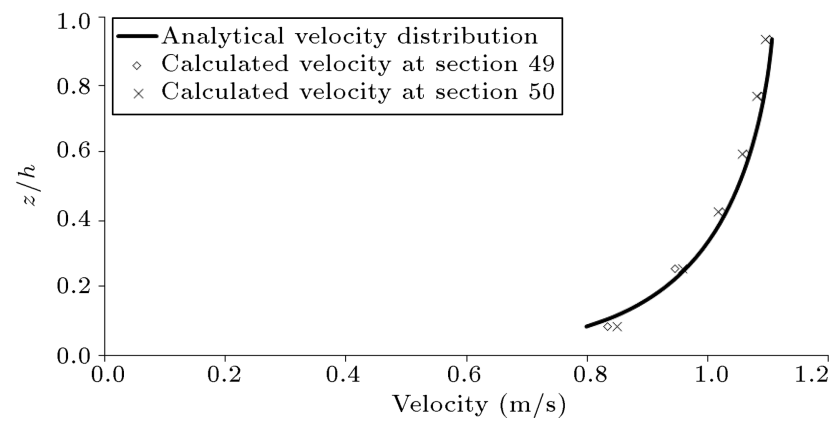

Figure 5. Oscillation of velocity profiles in two successive sections around the analytical result.

surface of the first layer (or bottom surface of the second layer) was positive and negative alternately. The calculation results showed that water surface elevation was oscillating around the correct answers (Figure 4). Also, the velocity distribution calculated by the model was oscillating around the analytical velocity distribution, in each of two successive sections (see Figure 5). Results, therefore, revealed that with a zigzag mesh the calculated velocity profiles and flow depth oscillated.

\subsection{Finding the origin of errors}

To find the reasons of oscillations, the discretized umomentum equation is studied in more detail. Consider a general layer-integrated $u$-momentum equation (Eq. (7)) for a uniform flow. The source term of this equation (Eq. (10)) can be simplified as follows:

$$
S r^{u}=-v_{t} \frac{\partial u}{\partial z}-u^{2} \frac{\partial z}{\partial x} .
$$

If $S r^{u}$ is substituted in the discretized $u$-momentum equation (Eq. (15)) based on the upwind scheme, this equation can be simplified and rearranged as follows:

$$
\begin{aligned}
a_{P} \bar{u}_{P}= & a_{W} \bar{u}_{W}+\left(-g h_{k} \frac{\partial \zeta}{\partial x}+\left.v_{t} \frac{\partial u}{\partial z}\right|_{z_{k}} ^{z_{k+1}}\right) \Delta x \Delta y \\
& +\left(\left.u^{2} \frac{\partial z}{\partial x}\right|_{z_{k}} ^{z_{k+1}}\right) \Delta x \Delta y, \quad \frac{\partial \zeta}{\partial x}=S_{0} .
\end{aligned}
$$

Here, $S_{0}$ is the bed slope. Since the first layer is consid- 
ered, $\left.v_{t} \frac{\partial u}{\partial z}\right|_{z_{k}}$ is calculated using the wall function as bed shear stress (see Section 1.4). In addition, $\left.u^{2} \frac{\partial z}{\partial x}\right|_{z_{k}}$ is zero. Eqs. (17) and (18) can be simplified in a uniform flow as follows:

$$
\begin{aligned}
& a_{P}=a_{W}+\left(F_{e}-F_{w}\right), \\
& F_{e}-F_{w}=\left.u \frac{\partial z}{\partial x}\right|_{z_{k}} ^{z_{k+1}} \Delta x \Delta y .
\end{aligned}
$$

Combining Eqs. (30) and (31) yields:

$$
\begin{aligned}
a_{W} \bar{u}_{P}= & a_{W} \bar{u}_{W}+\left(-g h_{k} \frac{\partial \zeta}{\partial x}+\left.v_{t} \frac{\partial u}{\partial z}\right|_{z_{k}} ^{z_{k+1}}\right) \Delta x \Delta y \\
& +\left(\left.u\left(u-\bar{u}_{P}\right) \frac{\partial z}{\partial x}\right|_{z_{k}} ^{z_{k+1}}\right) \Delta x \Delta y
\end{aligned}
$$

The first term in the bracket on the right hand side of the above equation represents forces on the mass in the computational cell (the hatched cell in Figure 6). Given that the velocity is constant along the flow direction in a uniform flow, the sum of all forces on any arbitrary control volume should be zero. Hence, Eq. (32) can be simplified as follows:

$$
a_{W} \bar{u}_{P}=a_{W} \bar{u}_{W}+\left(\left.u\left(u-\bar{u}_{P}\right) \frac{\partial z}{\partial x}\right|_{z_{k}} ^{z_{k+1}}\right) \Delta x \Delta y .
$$

Since the flow is uniform and the elevations of the center of all velocity cells along the flow direction are the same, $\bar{u}_{P}$ should be equal to $\bar{u}_{W}$ (Figure 6). However, the second term on the right hand side of Eq. (33) is a non-zero value in a non-rectangular mesh due to the existence of $\partial z / \partial x$ and becomes positive and negative in successive cells in the mesh layout of the present example. Therefore, the velocity of the two following cells increases and decreases, causing the flow depth to oscillate.

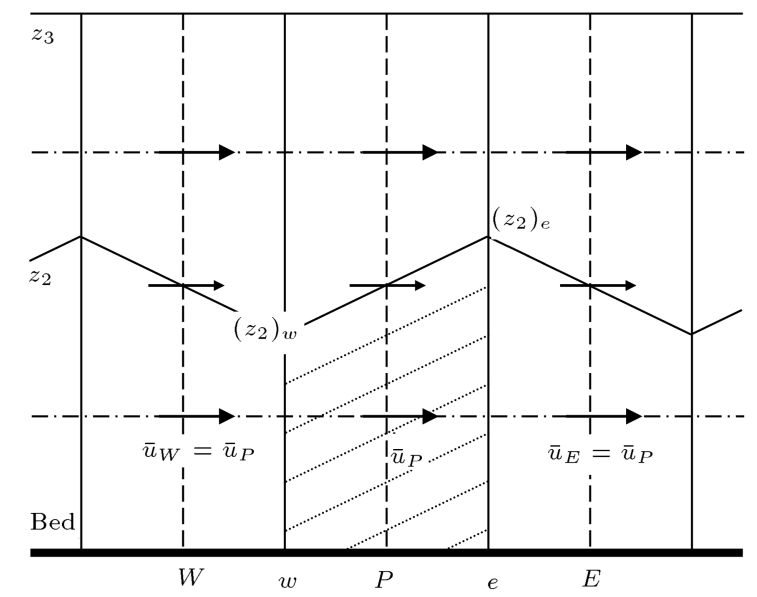

Figure 6. Location of velocity and velocity cells in the computational mesh in depth.

\subsection{The effect of differential advection terms}

In the process of deriving layer-integrated momentum equations, differential advection terms (Eqs. (13) and (14)) were eliminated. Elimination of these terms is equivalent to assuming constant velocity on the faces of the computational cells. This assumption is a usual practice in a finite volume method. It is shown that such an assumption is the reason for computational errors in a non-rectangular mesh. In a uniform flow, the following term is removed from the differential advection terms (Eqs. (13) and (14)):

$$
\frac{\partial}{\partial x} \int_{z_{k}}^{z_{k+1}}(u-\bar{u})(u-\bar{u}) d z .
$$

The above term can be simplified in the following form:

$$
\begin{aligned}
& \int_{z_{k}}^{z_{k+1}} u(u-\bar{u}) d z-\int_{z_{k}}^{z_{k+1}} \bar{u} u d z+\int_{z_{k}}^{z_{k+1}} \overline{u u} d z \\
& \quad=\int_{z_{k}}^{z_{k+1}} u(u-\bar{u}) d z-\bar{u} h_{k}+\overline{u u} h_{k}=\int_{z_{k}}^{z_{k+1}} u(u-\bar{u}) d z .
\end{aligned}
$$

Using the Leibnitz integration rule (Eq. (5)), one can write:

$$
\begin{aligned}
& \frac{\partial}{\partial x} \int_{z_{k}}^{z_{k+1}}(u(u-\bar{u})) d z=\int_{z_{k}}^{z_{k+1}} \frac{\partial}{\partial x}(u(u-\bar{u})) d z \\
& \quad+\left.u(u-\bar{u}) \frac{\partial z}{\partial x}\right|_{z_{k}} ^{z_{k+1}} .
\end{aligned}
$$

The first term on the right hand side of the above equation can be written as:

$$
\int_{z_{k}}^{z_{k+1}} \frac{\partial}{\partial x}(u(u-\bar{u})) d z=\int_{z_{k}}^{z_{k}+1}\left(\frac{\partial u^{2}}{\partial x}-\bar{u} \frac{\partial u}{\partial x}-u \frac{\partial \bar{u}}{\partial x}\right) d z .
$$

In a uniform flow $\partial u / \partial x$ and in the present situation in Figure 6, $\partial \bar{u} / \partial x$ is equal to zero. Consequently, all the terms in Eq. (37) (the first term on the right hand side of Eq. (36)) are equal to zero.

In the second term on the right hand side of Eq. (36), $\bar{u}$ is the layer-averaged velocity and is equal to $\bar{u}_{P}$ due to upwind scheme. Therefore, the second term on the right hand side of Eq. (36) can be written as $u\left(u-\bar{u}_{P}\right) \partial z /\left.\partial x\right|_{z_{k}} ^{z_{k+1}}$. This term is actually what remains from the differential advection terms for the uniform flow. By multiplying this term by $\Delta x \Delta y$ and adding it to the right hand side of Eq. (33) with a negative sign, the second term on the right hand side of this equation is canceled. In this way, by adding the differential advection term to the momentum equation, momentum fluxes in the cell are balanced. As a result, 
velocity remains constant along the flow direction and the problem of velocity oscillation is solved.

\subsection{Development of the model by adding differential advection terms}

In order to examine the effect of the differential advection terms, Eq. (34) was discretized and added to the source term of $u$-momentum equation as follows:

$$
\begin{aligned}
\int_{\mathrm{C.V} .} & \left(\frac{\partial}{\partial x} \int_{z_{k}}^{z_{k+1}}(u-\bar{u})^{2} d z\right) d V \\
= & {\left[\left(\int_{z_{k}}^{z_{k+1}}(u-\bar{u})^{2} d z\right)_{e}-\left(\int_{z_{k}}^{z_{k+1}}(u-\bar{u})^{2} d z\right)_{w}\right] \Delta y } \\
= & {\left[\int_{\left(z_{k}\right)_{e}}^{\left(z_{k+1}\right)_{e}}\left(u-\bar{u}_{e}\right)^{2} d z-\int_{\left(z_{k}\right)_{w}}^{\left(z_{k+1}\right)_{w}}\left(u-\bar{u}_{w}\right)^{2} d z\right] \Delta y }
\end{aligned}
$$

where $\bar{u}_{e}$ and $\bar{u}_{w}$ are the layer-averaged velocities on the east and west faces of the control volume, which are determined by interpolation from the neighboring velocity values. $u$ is the velocity value at different levels on these faces. To calculate the above integral, distribution of $u$ velocity should be known along the cell faces. For this purpose, the velocity values at the bottom, center, and top of each face were interpolated from the neighboring values. A linear distribution was then assumed for velocity in each layer.

Linear velocity distribution assumption is not appropriate for the bottom layer due to the high velocity gradient in this layer. Therefore, a logarithmic velocity distribution was employed at the bottom layer, similar to that used in a wall function.

The results showed that by adding the differential advection term to the model, oscillations of the velocity and water depth were eliminated. Figure 7 shows the water surface profile, before and after adding the differential advection term to the numerical model.

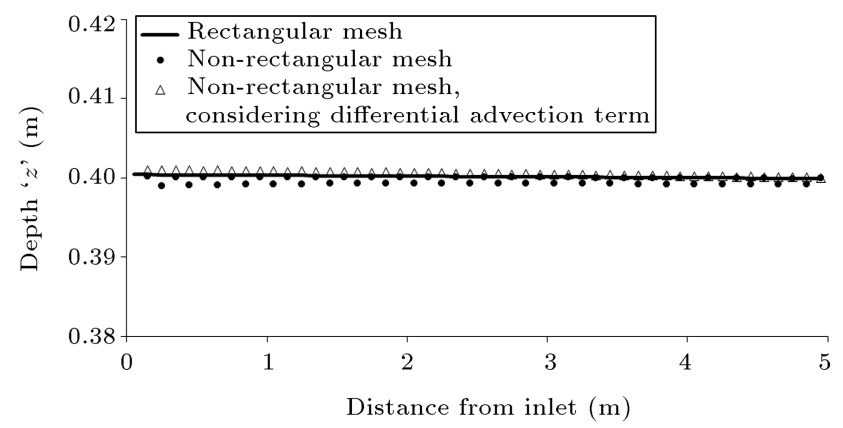

Figure 7. Water free surface elevation, before and after adding the differential advection term to the numerical model.

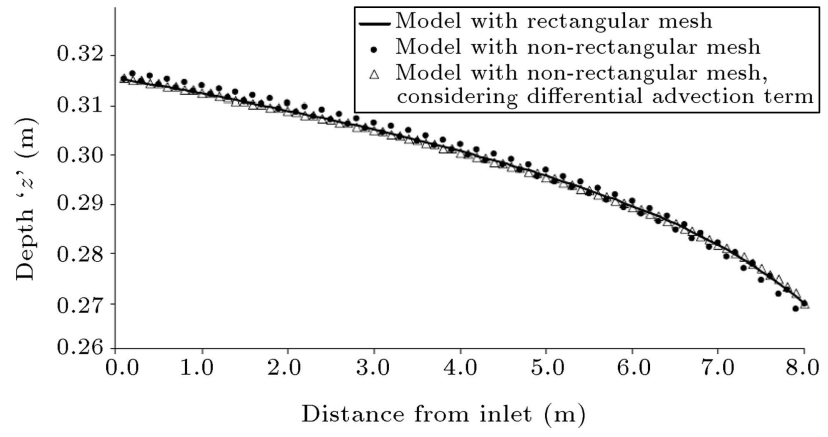

Figure 8. Water free surface elevation, before and after adding the differential advection term to the numerical model in a non-uniform flow.

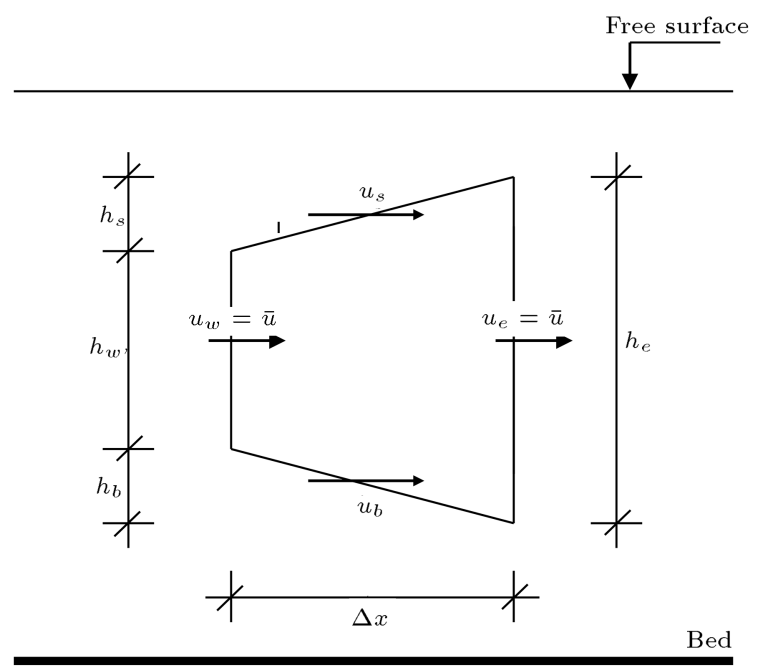

Figure 9. Non-rectangular control volume in a uniform flow.

The computed velocity distribution profiles were also enhanced.

An additional example was solved with a nonuniform flow. Figure 8 shows the results. According to this figure, using a zigzag mesh similar to that shown in Figure 3 caused oscillation in water surface. This oscillation, however, was eliminated as the differential advection term was added to the computer model (Figure 8).

\section{Extending the results to finite volume models}

In the previous section, by using the $3 \mathrm{D}$ shallow water model, it was shown that the oscillation of the results in a zigzag mesh condition was due to assuming constant velocity on the faces of computational cells. The same error, therefore, is expected in any finite volume model when non-rectangular computational cells are used. This issue is investigated in the following section.

A non-rectangular computational cell, with its longitudinal axis parallel to the bed, is assumed in a uniform flow, as shown in Figure 9. The slopes of 
the top and the bottom faces, relative to flow bed, are $\partial z /\left.\partial x\right|_{s}$ and $\partial z /\left.\partial x\right|_{b}$, respectively. Hence, we have:

$$
u_{e}=u_{w}=\bar{u}
$$

where $\bar{u}$ is the velocity value at the center of the east and west faces, $\Delta x$ and $\Delta y$ are the length and width of the cell, respectively, and $h_{e}, h_{w}, h_{b}$, and $h_{s}$ are the distances between the cell vertexes, as shown in Figure $8 . h_{b}$ and $h_{s}$ are equal due to symmetry of the cell. The momentum equation for this control volume can be written as follows:

$$
\sum F_{x}=\iint_{\mathrm{C} . \mathrm{S} .} \rho u\left(\overrightarrow{v_{r}} \cdot \vec{n}\right) d A
$$

In this equation, $\vec{n}$ is the unit vector of the control surface and $\overrightarrow{v_{r}}$ is the flow velocity vector relative to control volume. It should be noted that since the velocity is constant in the flow direction, the resultant of all forces on any arbitrary control volume should be equal to zero. Therefore, the summation of momentum fluxes in and out of the assumed control volume should be equal to zero. Eq. (40) can be integrated over the control volume surfaces, assuming constant $\rho$ and velocities on each face:

$$
u_{w} u_{w} h_{w} \Delta y-u_{e} u_{e} h_{e} \Delta y+u_{s} u_{s} h_{s} \Delta y+u_{b} u_{b} h_{b} \Delta y=0 .
$$

Since $h_{e}=h_{b}+h_{w}+h_{s}$, Eq. (41) can be written as follows:

$$
\begin{gathered}
u_{w} u_{w} h_{w} \Delta y-u_{e} u_{e} h_{b} \Delta y-u_{e} u_{e} h_{w} \Delta y-u_{e} u_{e} h_{s} \Delta y \\
+u_{s} u_{s} h_{s} \Delta y+u_{b} u_{b} h_{b} \Delta y=0
\end{gathered}
$$

The above equation could be rearranged as:

$$
\begin{aligned}
u_{e} u_{e} h_{w} \Delta y= & u_{w} u_{w} h_{w} \Delta y+\left(u_{s} u_{s}-u_{e} u_{e}\right) h_{s} \Delta y \\
& +\left(u_{b} u_{b}-u_{e} u_{e}\right) h_{b} \Delta y .
\end{aligned}
$$

Considering Eq. (39), substituting $u_{e} u_{e}=\overline{u u}$ on the right hand side of Eq. (43), and assuming $u_{e} h_{w} \Delta y=$ $u_{w} h_{w} \Delta y=a$, we have:

$$
a u_{e}=a u_{w}+\left(u_{s} u_{s}-\overline{u u}\right) h_{s} \Delta y+\left(u_{b} u_{b}-\overline{u u}\right) h_{b} \Delta y \text {. }
$$

Based on the above equation, if $u_{s}, u_{b}$, and $\bar{u}$ have different values, then $u_{e} \neq u_{w}$, which is in contrast with Eq. (39). This result indicates an error in the computation. It can be shown that Eq. (44) is the same as Eq. (33), which is the discretized momentum equation in a multi-layer model without differential advection terms and for the uniform flow condition. To prove this, one can write:

$$
h_{s}=\left.\frac{\partial z}{\partial x}\right|_{s} \Delta x, \quad h_{b}=-\left.\frac{\partial z}{\partial x}\right|_{b} \Delta x .
$$

If these two values are substituted in Eq. (44), then:

$$
\begin{aligned}
a u_{e}= & a u_{w}+\left.\left(u_{s} u_{s}-\overline{u u}\right) \frac{\partial z}{\partial x}\right|_{s} \Delta x \Delta y \\
& -\left.\left(u_{b} u_{b}-\overline{u u}\right) \frac{\partial z}{\partial x}\right|_{b} \Delta x \Delta y .
\end{aligned}
$$

On the other hand, the continuity equation can be discretized over the assumed control volume as:

$$
\bar{u} h_{w}-\bar{u} h_{e}+u_{s} h_{s}+u_{b} h_{b}=0 .
$$

Substituting $h_{e}=h_{b}+h_{w}+h_{s}$ in Eq. (47) results in:

$$
\bar{u} h_{w}-\bar{u} h_{b}-\bar{u} h_{w}-\bar{u} h_{s}+u_{s} h_{s}+u_{b} h_{b}=0 .
$$

Rearranging and multiplying the above equation in $\bar{u} \Delta y$ yields:

$$
\bar{u} \Delta y\left(\left(u_{s}-\bar{u}\right) h_{s}-\left(u_{b}-\bar{u}\right)\left(-h_{b}\right)\right)=0 .
$$

Substituting Eq. (45) in Eq. (49) yields:

$$
\left.\left(u_{s} \bar{u}-\overline{u u}\right) \frac{\partial z}{\partial x}\right|_{s} \Delta x \Delta y-\left.\left(u_{b} \bar{u}-\overline{u u}\right) \frac{\partial z}{\partial x}\right|_{b} \Delta x \Delta y=0
$$

If Eq. (50) is subtracted from the right hand side of Eq. (46), we have:

$$
a u_{e}=a u_{w}+\left(\left.u(u-\bar{u}) \frac{\partial z}{\partial x}\right|_{b} ^{s}\right) \Delta x \Delta y,
$$

which is similar to Eq. (33). Therefore, it can be concluded that assuming constant velocities on the faces of the computational cells causes inaccuracy with a non-rectangular mesh, similar to that encountered in the layer integrated model.

\section{Summary and conclusions}

Non-rectangular computational cells are generated for numerical simulation of fluid flows with irregular boundaries. In the present work, the effects of nonrectangular computational cells on the accuracy of numerical models were investigated. For this purpose, a $3 \mathrm{D}$ shallow water model, which was verified previously, was used to simulate a uniform flow with a zigzag computational grid. Results showed that with nonrectangular computational cells, the calculated velocity profiles and flow depth oscillated.

In order to determine the origin of these errors, the governing equations were carefully examined. In this examination, the discretized momentum equations were analyzed for a simple uniform flow. This analysis revealed that the source of error resulted from assuming constant velocity at computational cell faces, which is a usual assumption in finite volume models. The application of this assumption led to an imbalance in discretized momentum equations, 
causing error. It was also shown that assuming a velocity distribution on the cell faces reduced the errors. It was further shown that an error similar to that encountered in layer-integrated models could also occur in any finite volume numerical model when non-rectangular computational cells are used.

\section{Nomenclature}

a Coefficients of discretized equations

F Mass flux

$g \quad$ The gravitational acceleration

$h \quad$ Total depth

$h_{k} \quad k$ th layer thickness

$k_{s} \quad$ Absolute roughness

$M \quad$ Mass flux across the layer interfaces

$\vec{n} \quad$ Unit vector of the control surface

$n l \quad$ Number of layers

$P \quad$ Pressure

$S_{0} \quad$ Channel bed slope

Sr Source terms of the momentum equations

$S u \quad$ Mass residual

$u \quad$ Velocity component in $x$ direction

$\bar{u} \quad$ Layer averaged component of velocity in $x$ direction

$u_{*} \quad$ Bed shear velocity

$v \quad$ Velocity component in $y$ direction

$\bar{v} \quad$ Layer averaged component of velocity in $y$ direction

$\overrightarrow{v_{r}} \quad$ Flow velocity vector relative to control volume

$w \quad$ Component of velocity in $z$ direction

$z \quad$ Distance along the $z$ axis

$\Delta x, \Delta y \quad$ Mesh dimensions

$\kappa \quad$ Von Karman constant

$\rho \quad$ Density

$v_{t} \quad$ Eddy viscosity

$\zeta \quad$ Water surface elevation

\section{Superscripts}

* Value of quantity in the process of iteration

, Quantity correction

$u, v \quad$ Related to momentum equations in $x$ and $y$ directions, respectively

\section{Subscripts}

$k, k+1 \quad$ Bottom and top surfaces of a layer along the $z$ axis, respectively
$P, E, W$

$N, S$

$e, w, n, s \quad$ Positions on the faces of the scalar cells

nb Neighboring points

\section{References}

1. Vreugdenhil, C.B. and Wijbenga, J. "Computation of flow pattern in rivers", ASCE J. Hydraul. Div., 108(11), pp. 1296-1310 (1982).

2. Keller, R.J. and Rodi, W. "Prediction of flow characteristics in main channel/flood plain flows", J. Hydraul. Res., 26(4), pp. 425-441 (1988).

3. Lai, C.J. and Yen, C.W. "Turbulent free surface flow simulation using a multi-layer model", Int. J. Numer. Methods Fluids, 16, pp. 1007-1025 (1993).

4. Li, C.W. and Yu, T.S. "Numerical investigation of turbulent shallow recirculating flows by a quasi-threedimensional $k-\varepsilon$ model", Int. J. Numer. Methods Fluids, 23, pp. 485-501 (1996).

5. Ye, J. and McCorquodale, J.A. "Depth averaged hydrodynamic model in curvilinear collocated grid", ASCE J. Hydraul. Eng., 123(5), pp. 380-388 (1997).

6. Shankar, N.J., Chan, E.S., and Zhang, Q.Y. "Threedimensional numerical simulation for an open channel flow with a constriction", J. Hydraul. Res., 39(2), pp. 187-201 (2001).

7. Shao, X., Wang, H., and Chen, Z. "Numerical modeling of turbulent flow in curved channels of compound cross-section", Adv. Water Resour., 26(5), pp. 525-539 (2003).

8. Gholami, A., Akhtati, A.A., Minatour, Y., Bonakdary, H., and Javadi, A.A. "Experimental and numerical study on velocity field and water surface profile in a strongly-curved $90^{\circ}$ open channel bend", J. Eng. Appl. Comput. Fluid Mech., 8(3), pp. 447-461 (2014).

9. Yoshioka, H., Unami, K., and Fujihara, M. "A dual finite volume method scheme for catastrophic flash floods in channel networks", Appl. Math. Model., 39(1), pp. 205-229 (2015).

10. Zahraeifard, V. and Talebbeydokhti, N. "Numerical simulation of turbulent flow over labyrinth spillways/weirs and corresponding discharge coefficient and efficiency", Scientia Iranica, A, 22(5), 1734-1741 (2015).

11. Gholami, A., Bonakdari, H., Akhtari, A.A., and Ebtehaj, I. "A combination of computational fluid dynamics, artificial neural network and support vectors machines model to predict flow variables in curved channel", Scientia Iranica, A, 26(2), pp. 726-741 (2019).

12. Rezaei, B. and Amiri, H. "Numerical modeling of flow field in compound channels with non-prismatic floodplains", Scientia Iranica, A, 25(5), pp. 2413-2424 (2018). 
13. Tay, M.T.W., Mitchell, S.B., Chen, J., and Williams, J. "Numerical modelling approach for the management of seasonal influenced river channel entrance", Ocean Coast. Manag., 130, pp. 79-94 (2016).

14. Langendoen, E.J., Mendoza, A., Abad, J.D., et al. "Improved numerical modeling of morphodynamics of rivers with steep banks", Adv. Water Resour. Part A, 93, pp. 4-14 (2016).

15. Farmani, S., Barani, G., Ghaeini-Hessaroeyeh, M., and Memarzadeh, R. "Numerical modeling of flood waves in a bumpy channel with the different boundary conditions", Scientia Iranica, A, 26(2), pp. 667-677 (2019).

16. Peric, M. "Analysis of pressure velocity coupling on non-orthogonal grids", Numer. Heat Transfer, Part B, 17, pp. 63-82 (1990).

17. Zarrati, A.R. and Jin, Y.C. "Development of a generalized multi-layer model for 3-D simulation of free surface flows", Int. J. Numer. Methods Fluids, 46, pp. 1049-1067 (2004).

18. An, H. and Yu, S. "Finite volume integrated surfacesubsurface flow modeling on nonorthogonal grids", Water Resour. Res., 50, pp. 2312-2328 (2014).

19. Buitrago, S., Sosa, G., and Jimeénez, O. "An upwind finite volume method on non-orthogonal quadrilateral meshes for the convection diffusion equation in porous media", Appl. Anal., 95(10), pp. 2203-2223 (2016).

20. Shaw, J., Weller, H., Methven, J., and Davies, T. "Multidimensional method-of-lines transport for atmospheric flows over steep terrain using arbitrary meshes", J. Comput. Phys., 344, pp. 86-107 (2017).

21. Li, Y.S. and Zhan, J.M. "An efficient threedimensional semi-implicit finite element scheme for simulation of free surface flows", Int. J. Numer. Methods Fluids, 16, pp. 187-198 (1993).

22. Kim, C.K. and Lee, J.S. "A three-dimensional PCbased hydrodynamic model using an ADI scheme", Coast. Eng., 23, pp. 271-287 (1994).

23. Kodama, T., Wang, S.S.Y., and Kawahara, M. "Model verification on $3 \mathrm{D}$ tidal current analysis in Tokyo bay", Int. J. Numer. Methods Fluids, 22, pp. 43-66 (1996).

24. Wei, O.W.H., Lu, Q., and Li, Y.S. "Multi-layer modeling of three-dimensional hydrodynamic transport processes", J. Hydraul. Res., 34(5), pp. 677-693 (1997).
25. Reggio, M., Hess, A., and Ilinca, A. "3-D multiplelevel simulation of free surface flows", J. Hydraul. Res., 40(4), pp. 413-423 (2002).

26. Vreugdenhil, C.B., Numerical Methods for Shallow Water Flows, Kluwer Academic Publishers, Dordrecht (1994).

27. Patankar, S.V., Numerical Heat Transfer and Fluid Flow, Hemisphere Publishing corp., Washington, D.C. (1980).

28. Weerakoon, S.B., Tamai, N., and Kawahara, Y. "A depth correction equation for depth-averaged flow computations in natural rivers", Proceedings of the Environmental Hydraulics Conference, Hong Kong (1991).

29. Zhou, J.G. "Velocity-depth coupling in shallow water flows", ASCE J. Hydraul. Eng., 121(10), pp. 717-724 (1995).

\section{Biographies}

Masoud Morovvat did his BSc in Civil Engineering in Amirkabir University of Technology and his postgraduate studies on Water Engineering at Shahid Beheshti University. Mr. Morrovat is involved in various civil engineering projects after graduation.

Amir Reza Zarrati is a Professor of Hydraulic Engineering at Department of Civil and Environmental Engineering, Amirkabir Unversity of Technology. Dr Zarrati's main research interests are hydraulic structures, sediment transport, reservoir sedimentation, and scouring phenomenon and prevention all with using numerical methods and physical modeling. He is also cooperating with the water industry in designing hydraulic structures and river engineering works.

Mohammad Reza Jalili Ghazizadeh is an Associate Professor at the Faculty of Civil, Water, and Environmental Engineering, Shahid Beheshti University. He received his $\mathrm{PhD}$ in Water Civil Engineering in 2003 from Amirkabir University of Technology, Tehran, Iran. His research interests include water and wastewater networks, water demand management, hydraulic structures, and numerical methods with emphasis on their practical aspects. He has published more than 100 research papers. 